\title{
Ferramentas colaborativas e suas metodologias para tradutores e intérpretes de Libras: Uma Revisão Sistemática da Literatura
}

\author{
Ralfh Alan G. Machado ${ }^{1}$, Heleno Fülber ${ }^{1}$, Bruno Merlin ${ }^{1}$, Adonney Allan O. Veras ${ }^{1}$ \\ ${ }^{1}$ Programa de Pós-graduação em Computação Aplicada (PPCA) - Universidade Federal \\ do Pará (UFPA) - 68464-000 - Tucuruí - Pará - Brasil \\ \{ralfhalan, allanverasce\}@gmail.com,\{fulber, brunomerlin\}@ufpa.br
}

\begin{abstract}
The spread of information and communication technology (TIC) has revolutionized the way we share information. Collaborative tools are of great importance for group teaching and learning, as well as helping to disseminate knowledge. The translator and interpreter of Libras is the main agent in the teacher's communication with Deaf ${ }^{\prime}$ students. This research consists of a Systematic Review of Literature (RSL) of the last ten years that addresses these synergy resources developed for these professionals. We selected 12 articles for a more detailed analysis. The results show that the work related in the literature is still scarce.
\end{abstract}

Resumo. A propagação da tecnologia da informação e comunicação (TIC) tem revolucionado a maneira como compartilhamos informações. Ferramentas colaborativas são de grande importância para o ensinoaprendizagem em grupo, além de auxiliar na disseminação do conhecimento. O tradutor e intérprete de Libras é o principal agente na comunicação do professor com alunos Surdos ${ }^{1}$. Esta pesquisa consiste em uma Revisão Sistemática da Literatura (RSL) dos últimos dez anos que abordem esses recursos de sinergia desenvolvidos para esses profissionais. Foram selecionados 12 artigos para uma análise mais acentuada. Os resultados mostram que ainda são escassos os trabalhos relacionados na literatura.

\section{Introdução}

Segundo a lei $n^{\circ} 10.436$ [BRASIL 2002], a Libras (Língua Brasileira de Sinais) é reconhecida como meio legal de comunicação e expressão. O sistema educacional nas esferas federal, estadual, municipal e do Distrito Federal devem garantir a inclusão nos cursos de formação de educação especial, de fonoaudiologia e de magistério em seus níveis médio e superior, do ensino da Libras, como parte integrante dos parâmetros curriculares nacionais. Ressalta-se ainda que a inclusão permite que todo ser humano, naturalmente, possa participar de maneira plena e efetiva da sociedade que o cerca, em igualdade de oportunidades, sobrepondo-se a prática de exclusão manifestada de diversas maneiras no mundo atual.

\footnotetext{
1 Segundo pesquisas do americano James Woodward realizadas em 1972, existe uma diferença entre o surdo, com "s" minúsculo (não participa da comunidade surda, não usa a Libras) e o Surdo, com "S" maiúsculo (usa a Libras, participa ativamente da comunidade surda, tem sua cultura própria, não aceita ser tratado como um deficiente, mas sim como diferente).
} 
Os profissionais tradutores e intérpretes de Libras, em sua maioria são graduados em Letras-Libras, não tendo um conhecimento aprofundado nas mais diversas áreas do conhecimento, dificultando assim a transmissão do saber [De Lacerda 2010]. Outra importante questão é a escassez de sinais específicos, desse modo esse profissional fica impossibilitado de auxiliar o Surdo no processo de ensino das disciplinas especialistas, tendo que realizar a datilologia, que é a soletração de uma palavra utilizando o alfabeto digital ou manual da língua de sinais [De Quadros and Karnopp 2009]. Dessa maneira, esse cenário ocasiona que a comunicação e a transmissão do estudo se torne lenta e onerosa.

[Gonçalves et al. 2015] relatam que os alunos voltam a atenção durante a aula para o tradutor, pois é ele quem leva a comunicação do que está sendo ensinado. $\mathrm{O}$ trabalho desse profissional se apresenta como um grande desafio em diferentes áreas. Este especialista precisa cumprir diversas exigências, tais como: Domínio das línguas que traduz-interpreta, técnicas e modalidades de interpretação, história e cultura surda além do contato constante com a comunidade. Dessa forma, uma das estratégias para auxiliar o tradutor de Libras é o uso de ferramentas computacionais.

Ferramenta colaborativa computacional, software colaborativo ou groupware é um software delineado para apoiar os usuários envolvidos em tarefas ou objetivos comuns, fornecendo a interface para um ambiente compartilhado. A colaboração junto ao aprendizado virtual, diminuem as barreiras impostas pelo espaço físico e o tempo entre seus participantes, oferecendo formas de interação, controle, coordenação, cooperação e comunicação entre as partes envolvidas que compõem o grupo [Camargo et al. 2005].

Esse cenário motivou a realização de uma RSL para identificar nas principais bases de artigos científicos, trabalhos relacionados às ferramentas colaborativas e suas metodologias para os tradutores e intérpretes de Libras. O objetivo é categorizar e identificar os estudos relevantes nessa área e consequentemente, auxiliar a construção de novos mecanismos que venham ajudar no compartilhamento das experiências e do conhecimento.

O restante deste trabalho esta organizado da seguinte maneira: Na seção 2 abordamos a metodologia desta pesquisa, explicando todas as etapas seguidas no protocolo de revisão. Na seção 3 apresentamos os resultados obtidos após os critérios de inclusão e exclusão, assim como a síntese e análise dos artigos selecionados. Por fim, na seção 4 apresentamos as conclusões obtidas através desta revisão.

\section{Metodologia}

Como estratégia para verificar os estudos, as suas contribuições, bem como o estado da arte, utilizou-se a técnica de Revisão Sistemática (RS) para conduzir e alicerçar esse processo. A RS é uma técnica de pesquisa com base em evidências da literatura científica, conduzida por meio da definição de um processo e um protocolo de buscas bem definido [Biolchini et al. 2005]. As fases desse processo conforme [Kitchenham 2004] são: Planejamento, execução e a fase de sumarização dos resultados (figura 1). 
VIII Congresso Brasileiro de Informática na Educação (CBIE 2019)

Anais do XXX Simpósio Brasileiro de Informática na Educação (SBIE 2019)

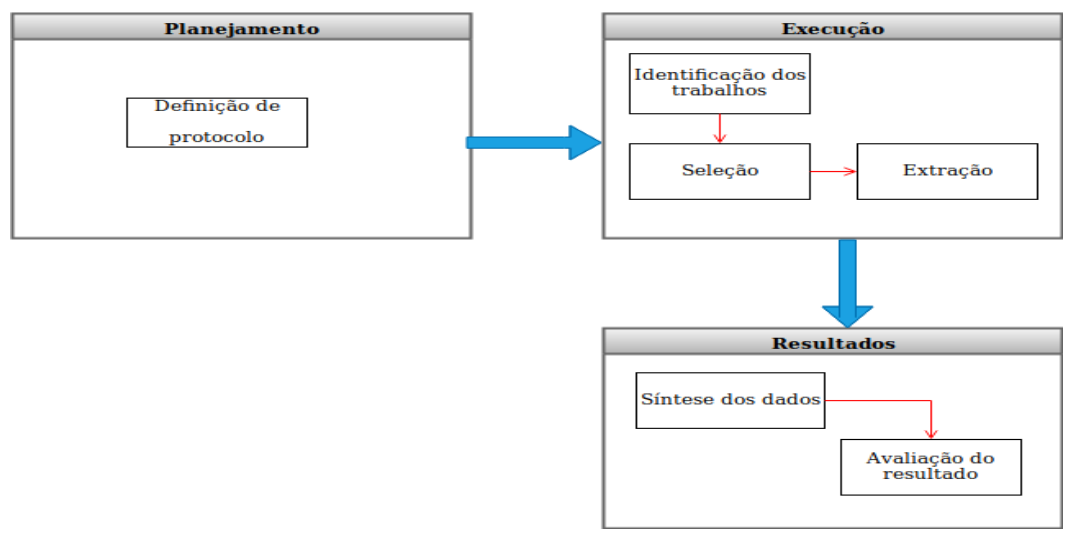

Figura 1. Fases de uma RSL - adaptado de [Vieira et al. 2016]

\subsection{Planejamento}

A primeira fase da RS é o planejamento que consiste na definição do protocolo que será seguido durante o processo da revisão. Nessa fase são definidas as questões de pesquisa, a string de busca, as fontes de pesquisa, os critérios de inclusão e exclusão, entre outros. As questões primárias de pesquisa que nortearam a realização desse trabalho foram as seguintes:

- QP1: Quais as ferramentas colaborativas disponíveis na literatura para tradutores e intérpretes de Libras?

- QP2: Quais as metodologias adotadas no desenvolvimento dessas ferramentas?

Para obtenção das respostas das questões de pesquisas (QP) levantadas, foi realizada uma busca nas principais bases de pesquisas científicas e informática aplicada a educação, considerando o período de 2009 a 2019. Essas bibliotecas permitem consultas online por meio de mecanismos de busca nas quais é possível utilizar expressões lógicas para definir a string de busca além de filtrar pelo ano de publicação. Determinou-se que somente os trabalhos nos idiomas inglês e português seriam considerados nesta revisão. As revistas e conferências selecionadas são apresentadas na Tabela 1.

Tabela 1. Fontes nacionais e internacionais pesquisadas

\begin{tabular}{|c|c|}
\hline Fonte de pesquisa & Acrônimo \\
\hline Revista Brasileira de Informática Brasileira na Educação & RBIE \\
\hline Simpósio Brasileiro de Informática na Educação & SBIE \\
\hline Workshop de Informática na Escola & WIE \\
\hline $\begin{array}{c}\text { Anais dos Workshops do Congresso Brasileiro de Informática na } \\
\text { Educação }\end{array}$ & WCBIE \\
\hline Revista de Informática Teórica e Aplicada & RITA \\
\hline Workshop sobre Educação em Computação & WEI \\
\hline Institute of Electrical and Electronics Engineers & IEEE Xplore Digital Library \\
\hline Association for Computing Machiner & ACM Digital Library \\
\hline Springer Link & - \\
\hline
\end{tabular}


VIII Congresso Brasileiro de Informática na Educação (CBIE 2019)

Anais do XXX Simpósio Brasileiro de Informática na Educação (SBIE 2019)

A string de busca foi gerada a partir da combinação das palavras-chave das QPs e foram divididas da seguinte forma (tabela 2):

Tabela 2. Strings de busca para a seleção de artigos

\begin{tabular}{|c|c|}
\hline Conceitos & Termos alternativos e sinônimos \\
\hline Ferramenta colaborativa & (("tool" OR "software" OR "collaborative" OR "participatory") \\
\hline \multicolumn{2}{|c|}{ AND } \\
\hline Tradutor e intérprete & ("translator" OR "interpreter") \\
\hline \multicolumn{2}{|c|}{ AND } \\
\hline Libras & ("sign language" OR "libras")) \\
\hline
\end{tabular}

Para as bases nacionais foram retirados os AND's da string, pois os mesmos são implícitos em tais engines. A tabela 3 apresenta os critérios de inclusão e exclusão que foram construídos considerando as questões de pesquisa.

Tabela 3. Critérios de inclusão e exclusão

\begin{tabular}{|c|c|c|}
\hline Critérios & ID & Descrição \\
\hline \multirow{2}{*}{ Inclusão } & 11 & Aborda ferramentas colaborativas para tradutores e intérpretes de Libras \\
\hline & 12 & Aborda metodologias utilizadas na construção de ferramentas colaborativas \\
\hline \multirow{6}{*}{ Exclusão } & E1 & Texto completo não disponível para acesso na Web \\
\hline & E2 & Publicações que não satisfaçam a nenhum critério de inclusão \\
\hline & E3 & Publicações em que o idioma seja diferente do exigido \\
\hline & E4 & Publicações de artigos duplicados \\
\hline & E5 & Publicações que não apresentem os termos da string de busca \\
\hline & E6 & $\begin{array}{l}\text { Publicações que não tenham disponibilidade de conteúdo para leitura e } \\
\text { análise dos dados }\end{array}$ \\
\hline
\end{tabular}

\section{Execução}

A segunda fase da RS conforme a figura 1, consiste na identificação dos trabalhos, seleção e extração. A identificação buscou selecionar todos os artigos científicos relevantes, relacionados à(s) questão(ões) de pesquisa; a seleção realizou a leitura dos resumos das publicações coletadas e elegeu aquelas que satisfaziam aos critérios determinados (inclusão/exclusão); a extração analisou os artigos selecionados na etapa anterior por meio da representação de suas informações através de tabelas e gráficos buscando unificar os resultados individuais de cada publicação utilizando técnicas estatísticas.

- A identificação dos trabalhos trouxe um total de 78 trabalhos.

- Na seleção, 58 trabalhos foram excluídos por não estarem ligados ao tema (E2) e 3 foram excluídos por estarem duplicados (E4), restando 17 artigos.

- Na extração, 3 trabalhos foram removidos por não terem disponibilidade para leitura e análise dos dados (E6) e outros 2 pelo idioma ser diferente do exigido (E3), restando 12 artigos incluídos. 
VIII Congresso Brasileiro de Informática na Educação (CBIE 2019)

Anais do XXX Simpósio Brasileiro de Informática na Educação (SBIE 2019)

A tabela 4 mostra o quantitativo de artigos selecionados nas etapas da fase de execução.

Tabela 4. Resultado geral nas bases de dados consultadas

\begin{tabular}{|c|c|c|c|c|c|}
\hline Fonte & $\begin{array}{c}\text { Total } \\
\text { retornado }\end{array}$ & Identificação & Seleção & Extração & $\begin{array}{c}\text { Total } \\
\text { incluído }\end{array}$ \\
\hline RBIE & 117 & 7 & 1 & 1 & 1 \\
\hline SBIE & 215 & 14 & 2 & 2 & 2 \\
\hline WIE & 123 & 5 & 1 & 1 & 1 \\
\hline WCBIE & 127 & 9 & 1 & 1 & 1 \\
\hline RITA & 41 & 0 & 0 & 0 & 0 \\
\hline WEI & 15 & 1 & 0 & 0 & 0 \\
\hline IEEE Xplore Digital Library & 25 & 13 & 2 & 1 & 1 \\
\hline ACM Digital Library & 37 & 11 & 7 & 4 & 4 \\
\hline Springer Link & 35 & 18 & 3 & 2 & 2 \\
\hline Total & 735 & 78 & 17 & 12 & 12 \\
\hline
\end{tabular}

Na Figura 2 ilustra-se a participação em porcentagem dos artigos selecionados por base de dados em que foram encontrados, incluídos para extração de dados. Somente as fontes com trabalhos selecionados para extração de dados estão presentes no gráfico.

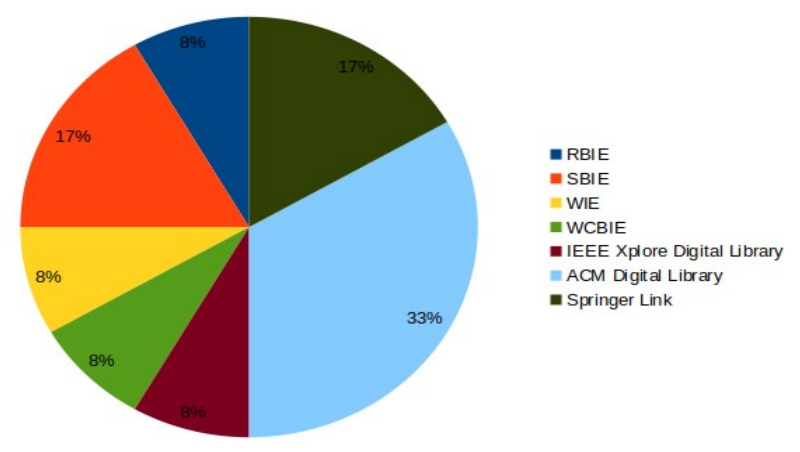

Figura 2: Porcentagem de participação dos artigos incluídos para extração de dados.

\subsection{Resultados e análises}

Esta seção demonstra os resultados produzidos pela extração de dados dos estudos primários de acordo com o processo descrito na etapa de extração assim como são apresentadas as respostas das questões de pesquisa definidas no protocolo desta revisão.

\section{QP1: Quais as ferramentas colaborativas disponíveis na literatura para tradutores e intérpretes de Libras?}

Foi possível observar, por meio das tecnologias encontradas que a maioria das publicações, tanto nacionais quanto internacionais, focam na construção colaborativa de sinais ou no ensino-aprendizagem de Libras. 
VIII Congresso Brasileiro de Informática na Educação (CBIE 2019)

Anais do XXX Simpósio Brasileiro de Informática na Educação (SBIE 2019) estudados.

A Tabela 5 mostra os grupos de características encontrados nos trabalhos

Tabela 5. Características das ferramentas

\begin{tabular}{|c|c|c|c|}
\hline ID & Grupos de Características & Artigo & $\begin{array}{c}\text { Número } \\
\text { de } \\
\text { trabalhos }\end{array}$ \\
\hline \multirow{4}{*}{ C1 } & \multirow{4}{*}{ Construção colaborativa de sinais } & [Silva et al. 2012] & \multirow{4}{*}{4} \\
\hline & & [latskiu et al. 2014] & \\
\hline & & [Jemni and Elghoul 2008] & \\
\hline & & [Rocha et al. 2015] & \\
\hline $\mathrm{C} 2$ & Compartilhamento de informações & [Da Silva and Prietch 2016] & 1 \\
\hline $\mathrm{C} 3$ & Interpretação colaborativa de sinais & [Rusňák et al. 2016] & 1 \\
\hline \multirow{6}{*}{ C4 } & \multirow{6}{*}{ Ensino-aprendizagem } & [De Araujo et al. 2016] & \multirow{6}{*}{6} \\
\hline & & [Lima and Araújo 2018] & \\
\hline & & [Reinoso et al. 2016] & \\
\hline & & [Reinoso and Tavares 2015] & \\
\hline & & [latskiu et al. 2018] & \\
\hline & & [Brandão et al. 2016] & \\
\hline
\end{tabular}

- FlexLibras [Silva et al. 2012]: Uma linguagem formal para descrever e animar sinais em Libras, permite a descrição de fonemas que representem um sinal.

- CPML [Iatskiu et al. 2014]: Interpretador que através do SignWriting (notação de escrita gráfica das linguagens gestuais), aborda uma nova maneira de gerar símbolos gráficos dos sinais de Libras.

- WebSign [Jemni and Elghoul 2008]: O sistema realiza uma tradução de texto escrito para a linguagem de sinais.

- AssistLIBRAS [Rocha et al. 2015]: Ferramenta onde especialistas podem criar os sinais, a um custo (tempo e esforço) menor.

- Wikitas [Da Silva and Prietch 2016]: Aplicação para compartilhar informações sobre tecnologias assistivas para pessoas Surdas.

- CoUnSiL [Rusňák et al. 2016]: Projeto de um ambiente de videoconferência para interpretação remota da linguagem de sinais.

- [De Araujo et al. 2016]: Solução baseada na plataforma Android, acessível e integrada com um Sistema de Gestão de Aprendizagem Acessível (ALMS).

- DINF [Lima and Araújo 2018]: Plataforma Web colaborativa com um dicionário contextualizado para o ensino de termos da área de informática em Libras.

- [Reinoso et al. 2016]: Plataforma para criação e uso de arquiteturas pedagógicas para aprendizagem de Libras.

- MVLIBRAS [Reinoso and Tavares 2015]: Ambiente digital de aprendizagem 
VIII Congresso Brasileiro de Informática na Educação (CBIE 2019)

Anais do XXX Simpósio Brasileiro de Informática na Educação (SBIE 2019)

colaborativa na qual visa o aprendizado de Libras e a língua portuguesa.

- [Iatskiu et al. 2018]: Sistema que permite a geração automática de sinais a partir de sua especificação num modelo fonológico.

- SlidePacer [Brandão et al. 2016]: Ferramenta que promove a coordenação entre instrutores e intérpretes de sinais.

\section{QP2: Quais as metodologias adotadas no desenvolvimento dessas ferramentas?}

Em [Silva et al. 2012], [Iatskiu et al. 2014] e [Jemni and Elghoul 2008] utilizam como base o XML (Extensible Markup Language), no primeiro trabalho é criada uma linguagem na qual um sinal é definido através de um conjunto de parâmetros que são representados por um avatar 3D. A mesma define amplas classes de fonemas e parâmetros tornando-a mais flexível, para a criação do avatar é necessário definir a localização e rotação dos 15 ossos localizados na mão. Almeja-se com o estudo a possibilidade de desenvolver ferramentas de realidade virtual para o ensino de Libras. $\mathrm{O}$ segundo trabalho tem como base dois modelos de arquitetura, uma em três camadas e a outra uma funcional integrada na qual se obtém uma descrição de um sinal de acordo com o modelo de fonologia e produz o conjunto correspondente de componentes do SignWriting. Em conjunto com esta arquitetura, foi desenvolvido um Modelo da Fonologia de Libras (CMPL) que apoia o desenvolvimento dos módulos da arquitetura funcional como parte do processamento da língua portuguesa com a de sinais. $\mathrm{O}$ trabalho propõe uma saída alternativa de escrita para o Português com um módulo tradutor. No terceiro trabalho é definida uma aplicação Web baseada na tecnologia de avatar 3D usando SML (linguagem descritiva que suporta uma abordagem colaborativa baseada em XML), o sistema utiliza a Xerox Web-Service para segmentação de uma frase e retorna o conjunto de palavras com sua categoria gramatical. A interface do sistema permite modificar o estado ou a posição do avatar e mostrar em tempo real o resultado da modificação no personagem 3D.

[Rocha et al. 2015] construíram a ferramenta sob forma de interface projetada no software 3D Studio Max que abstrai detalhes do usuário, como interpolação de forma e movimento, ou seja, o usuário pode selecionar o membro do personagem e mover para o local desejado, movendo também o marcador da linha de tempo. As interações se dão através de interfaces, não sendo necessário ter conhecimentos de programação, utiliza-se um arquivo com layout e configurações fixas, próprio para a criação dos sinais. A ferramenta permite que o especialista gere seus próprios sinais. [Rusňák et al. 2016] definem um sistema cliente-servidor que usa o UltraGrid para transmissões de áudio e vídeo de baixa latência e o middleware CoUniverse para orquestrar ambientes complexos colaborativos multi-ponto. Assim, é construído um plano de controle independente de transmissões de dados, um dos nós no ambiente, chamado grupo de aplicativos controller, centraliza a informação dos outros nós e decide sobre a estrutura de distribuição de dados. O trabalho ajuda intérpretes na tradução de cursos especializados.

Em [Da Silva and Prietch 2016] a aplicação baseia-se no conceito de Wiki, no sentido de permitir que os usuários contribuam e editem informações, sugiram novos materiais ou, ainda, descrevam experiências de uso de uma determinada tecnologia assistiva disponibilizada. A prototipação foi realizada utilizando a Unified Modeling 
VIII Congresso Brasileiro de Informática na Educação (CBIE 2019)

Anais do XXX Simpósio Brasileiro de Informática na Educação (SBIE 2019)

Language (UML) e uma avaliação junto ao publico alvo. As etapas foram definidas pensando no desenvolvimento de Projeto Centrado no Usuário (PCU) e na Experiência do Usuário (UX). [De Araujo et al. 2016] desenvolveram um aplicativo nativo na plataforma Android utilizando serviços via REST. A interface do usuário suporta requisitos de acessibilidade, tais como: Comandos de voz, definir a posição dos botões, configuração do tamanho da fonte e alto-contraste. O desenvolvimento também utilizou o conceito de prototipação e desenvolvimento iterativo coletando os requisitos de diversos profissionais. Espera-se que com a solução oferecendo chats e fóruns, facilite o ensino-aprendizagem.

[Lima and Araújo 2018] construíram a ferramenta apoiada pelo sistema de gerenciamento de conteúdo WordPress, a plataforma possui uma interface de uso simples com apenas um campo para a busca da palavra desejada e alguns botões de compartilhamento para incentivar a divulgação no círculo social do estudante. $\mathrm{O}$ dicionário pretende facilitar o entendimento de termos relacionados com a área de informática. [Iatskiu et al. 2018] criaram uma arquitetura computacional para a Interação Humano-Computador em Língua de Sinais (HCI-SL). Essa arquitetura possui um modulo central, o CORE-SL - modelo computacional para representação de sinais em uma arquitetura de serviços HCI-SL que tem a capacidade de representar, em princípio, os sinais de qualquer língua de sinais existente por meio de um formalismo. Espera-se proporcionar a base para o desenvolvimento de ferramentas para a construção de aplicações em línguas de sinais.

[Reinoso et al. 2016] utilizam o MOrFEu (Multi-Organizador Flexível de Espaços Virtuais) um modelo para a criação de sistemas e produção de espaços virtuaisdigitais de ensino. Realizaram um estudo etnográfico a partir de análises de documentos institucionais, em seguida, a especificação de requisitos do sistema, a análise e o projeto da plataforma CAP (Construtor de Arquiteturas Pedagógicas), assim como a modelagem do banco de dados, as funcionalidades da plataforma e a implementação do protótipo. A plataforma alcançou índices de usabilidade e funcionalidade aceitáveis. [Reinoso and Tavares 2015] desenvolveram um ADA (Ambiente Digital de Aprendizagem) baseado em arquiteturas pedagógicas e recursos digitais para aprendizagem colaborativa de Português e Libras. O sistema permite a construção individual de um dicionário de gestos visio-espaciais além de grupos de dicionários pelos intérpretes. Esse recurso permite registrar sinais usados pelos aprendizes para acompanhar sua evolução, bem como o registro de expressões locais em um dicionário comum para um grupo de pessoas.

[Brandão et al. 2016] agregam em um único ambiente a entrega de conteúdo, permitindo que o aluno Surdo leia as informações de slides reduzindo o atraso entre instrutor e intérprete. Foi implementado um componente instrutor como um pluggin do PowerPoint que possibilita a conexão do mesmo através de Bluetooth para que seja realizada a integração dos dois profissionais. O ambiente do intérprete consiste em um aplicativo Smartwatch móvel e um acompanhante que utiliza feedback visual e vibrotátil para informar aos intérpretes que uma ação é necessária, seja uma troca de slide pelo apresentador, ou mesmo a notificação que um aluno concluiu a interpretação.

Como resultado da QP2, observa-se que diferentes abordagens e metodologias foram utilizadas na construção dessas ferramentas, mesmo as de características em comum. Alguns trabalhos tendem a utilizar a tecnologia XML e a geração de avatares 
VIII Congresso Brasileiro de Informática na Educação (CBIE 2019)

Anais do XXX Simpósio Brasileiro de Informática na Educação (SBIE 2019)

3D, notou-se também a predominância pelos sistemas Web, evidenciando a carência de ferramentas para outras plataformas. Evidencia-se que alguns trabalhos definiram sua metodologia de maneira superficial ou incompleta, o que dificultou uma análise mais profunda e uma melhor sistematização dos resultados. Além disso, pelo fato de focar na interatividade desses profissionais através de chats, fóruns e Wikis, destaca-se ainda que as ferramentas mais adequadas dentre as recuperadas no trabalho foram: [Da Silva and Prietch 2016] e [De Araujo et al. 2016].

\section{Conclusão e considerações finais}

O mapeamento sistemático apresentado neste trabalho permitiu analisar as ferramentas colaborativas e suas metodologias para tradutores e intérpretes de Libras. Com a análise dos dados extraídos foi possível observar uma escassez de trabalhos nos quais o foco fosse de maneira mais acentuada nesse profissional.

Conclui-se ainda que a maioria das ferramentas visam a construção colaborativa de sinais ou ensino-aprendizagem de Libras. A partir disso, abre-se a oportunidade de construção de ferramentas com objetivos mais amplos e que visem auxiliar diretamente esse profissional.

Como trabalhos futuros, sugere-se a criação de ferramentas com o objetivo principal de qualificar através da socialização do conhecimento, permitindo assim a interatividade no acesso a informações, tendo em vista que esse profissional não tem formação específica nas diferentes áreas do saber e que, junto ao professor, é peça central no processo de transmissão do conhecimento para os Surdos.

\section{Referências}

Biolchini, Jorge, et al. (2005) "Systematic review in software engineering." System Engineering and Computer Science Department COPPE/UFRJ, Technical Report ES 679.05: 45 .

Brandão, Alessandra, et al. (2016) "SlidePacer: Apresentation delivery tool for instructors of deaf and hard of hearing students." Proceedings of the 18th International ACM SIGACCESS Conference on Computers and Accessibility. ACM.

BRASIL. Lei $\mathrm{n}^{\circ}$ 10.436, de 24 de abril de 2002, que dispõe sobre a Língua Brasileira de Sinais - Libras Disponível em: <http://www.planalto.gov.br/ccivil 03/leis/2002/ 110436.htm >. Acesso em: 24/02/2019 as 18:00 h.

Camargo, Á. A. B. De.,Khouri, L. H. El e Giarola, P. C. (2005) O Uso de Sistemas Colaborativos na Gestão de Projetos: Fatores Relevantes para o Sucesso. Trabalho de Conclusão de Curso. Fundação Instituto de Administração - FIA.

Da Silva, Wanderson Rodrigues, and Soraia Silva Prietch. (2016) "Wikitas: Online Collaboration concerning with Assistive Technologies for Teachers of Students who are Deaf." Proceedings of the 15th Brazilian Symposium on Human Factors in Computing Systems. ACM.

De Araujo, Thiago Alves Costa, et al. (2016) "Android accessible m-learning application for people with hearing and physical disabilities." International 
VIII Congresso Brasileiro de Informática na Educação (CBIE 2019)

Anais do XXX Simpósio Brasileiro de Informática na Educação (SBIE 2019)

Conference on Human-Computer Interaction. Springer.

De Lacerda, Cristina Broglia Feitosa. (2010) "Tradutores e intérpretes de Língua Brasileira de Sinais: Formação e atuação nos espaços educacionais inclusivos." Cadernos de Educação 36.

De Quadros, Ronice Müller, and Lodenir Becker Karnopp. (2009) Língua de sinais brasileira: estudos lingüísticos. Artmed Editora.

Gonçalves, E. J. T., Oliveira, F., Vilela, J. F., Peixoto, M. M., and Castro, J. B. (2015). "Produção de videoaulas de programação em java acessíveis no contexto de um projeto de capacitação profissional para pessoas surdas". Anais do XXVI SBIE.

Iatskiu, Carlos EA, et al. (2014) "A CPML-SignWriting Interpreter: A New Form to Generate the Graphical Symbols of SignWriting." International Conference on Universal Access in Human-Computer Interaction. Springer.

Iatskiu, Carlos Eduardo, et al. (2018) "Sistema para a Geração Automática da Escrita de Sinais em SignWriting visando o Apoio ao Ensino e à Aprendizagem da Libras." Brazilian Symposium on Computers in Education (Simpósio Brasileiro de Informática na Educação-SBIE). Vol. 29. No. 1.

Jemni, Mohamed, and Oussama Elghoul. (2008) "A system to make signs using collaborative approach." International Conference on Computers for Handicapped Persons. Springer, Berlin, Heidelberg.

Kitchenham, B. (2004). Procedures for performing systematic reviews. Keele, UK, KeeleUniversity, 33 (2004):1-26.

Lima, Geycy, and Rafael Araújo. (2018) "DINF: Uma Plataforma Online para Termos da Área de Informática em Libras." Anais do Workshop de Informática na Escola. Vol. 24. No. 1.

Reinoso, Luiz, and Orivaldo Tavares. (2015) "MVLIBRAS: Ambiente digital para comunidades de aprendizagem com recursos inclusivos para surdos." Brazilian Symposium on Computers in Education (Simpósio Brasileiro de Informática na Educação-SBIE). Vol. 26. No. 1.

Reinoso, Luiz, Renan Almeida, and Orivaldo Tavares. (2016) "Uma plataforma para construção de arquiteturas pedagógicas para a aprendizagem de LIBRAS." Anais dos Workshops do Congresso Brasileiro de Informática na Educação. Vol. 5. No. 1.

Rocha, Denys Fellipe Souza, I. B. Santana Pinto, and R. A. Silva. (2015) "AssistLIBRAS: Uma Ferramenta de Autoria para a Construção de Sinais da LIBRAS." Revista Brasileira de Informática na Educação 23.02:190.

Rusňák, Vít, et al. (2016) "CoUnSiL: Collaborative Universe for Remote Interpreting of Sign Language in Higher Education." International Conference on Computers Helping People with Special Needs. Springer.

Silva, Danilo Assis Nobre dos S., et al. (2012) "A formal language to describe and animate signs in brazilian sign language." SBC 3.2: 17.

Vieira, M. A. et al. (2016). Modelagem de espaços inteligentes pessoais e espaços inteligentes fixos no contexto de cenários de computação ubíqua. 